\title{
Comparative analysis and antimicrobial action of some essential oils from plants
}

\author{
Monica Mironescu ${ }^{1 *}$, and Cecilia Georgescu ${ }^{1}$ \\ ${ }^{1}$ Faculty of Agricultural Sciences Food Industry and Environmental Protection, Research Centre in Biotechnology and Food \\ Engineering, University Lucian Blaga of Sibiu Sibiu, Romania
}

\begin{abstract}
In this research, essential oils (EOs) were extracted through steaming from aerial parts of three plants: basil (Ocinum basilicum L.), peppermint (Mentha piperita L.) and oregano (Origanum vulgare L.) form Sibiu County, Romania. The GC-MS analysis indicated that eugenol and apiol were the major compounds in the basil EO. Timol, y-terpinene, and p-cymene were the major compounds in the volatile oil extracted from oregano and menthol and methone the main components in the peppermint EO. In the first $48 \mathrm{~h}$ of analysis, basil EO inhibition action was the highest against Salmonella anatum (48\%), followed by Aspergillus niger (26\%), Bacillus cereus (21\%) and Saccharomyces cerevisiae (15\%); this action was maintained for $120 \mathrm{~h}$ for all samples. In the first $48 \mathrm{~h}$, the peppermint EO showed the highest inhibition rate $(50 \%)$ on B. cereus, followed by A. niger (45\%), S. cerevisiae $(20 \%)$ and no action against Salmonella; this action varied after $72 \mathrm{~h}$ and $120 \mathrm{~h}$ by slightly increasing in case of B. cereus, strongly decreasing in case of A. niger and remaining constant in case of S. cerevisiae. Oregano EO showed a very small antimicrobial action, only on S. anatum (12\%) and this action was maintained for $120 \mathrm{~h}$.
\end{abstract}

\section{Introduction}

Aromatic plants are rich sources of bioactive compounds or bio nutrients [1]. Their essential oils (EOs) are final products of plants metabolism, accumulated and stored in various plant organs, in cells with intracellular secretion or in secretory tissues. Essential oils are natural, volatile complex compounds characterized by the odor of their corresponding aromatic plants, which synthesize them as secondary metabolites $[2,3]$. They have a lot of applications in food industry, cosmetics, perfumeries and pharmacy industry, due to their powerful antimicrobial and antioxidant activity [4]. The benefits resulting from the use of natural products rich in bioactive substances have promoted the growing interest of pharmaceutical, food and cosmetic industries as well as of individual consumers in the quality of herbal products $[5,6]$.

The EOs are complex mixtures that may contain over 300 different compounds [7]. Essential oils are natural mixtures of hydrocarbons (terpenes), oxygen-(alcohols, aldehydes, ketones, carboxylic acids, esthers, lactones) and sulphur-containing (sulphides, disulphides, trisulphides) organic substances. They are obtained mostly from plants by steam distillation or by extraction with carbon dioxide $[8,9]$.

Many articles present studies on volatile oils extracted from basil, peppermint or oregano $[10,11,12]$. Peppermint EO is one of the most usually used volatile oil in food and beverages, whereas basil and oregano oil are used in Mediterranean-origin food and in pharmaceutical and cosmetic market segments.

Peppermint (Mentha piperita L.) essential oil is usually used topically as antimicrobial, antipruritic, antiseptic, rubefacient and astringent as well as for healing headaches, neuralgia, migraines and myalgia [13]. Basil (Ocimum basilicum L.) is an annual plant found in the wild tropical, subtropical and temperate regions of the world. It takes part from Labiatae (Lamiaceae) family. Basil is a common herb, grown also in many households with a broad range of therapeutic properties; it has reputed medicinal uses as antioxidant, antibacterial, antimicrobial, antifungal, antiviral, cytoprotective, anticonvulsant, hypoglycaemic, hypolipidemic, hepatoprotective, renoprotective, neuroprotective, spermicidal, dermatologic and insecticidal [14]. Oregano (Origanum vulgare L.) EO have been shown to possess antioxidant, antibacterial, antifungal, diaphoretic, carminative, antispasmodic and analgesic activities and, among these, the antimicrobial potential is of special interest $[15,16]$.

The aim of this study is to compare the composition and the antimicrobial activity of EOs extracted from aerial parts of the three plants considered (basil, peppermint and oregano) against microorganisms possible to poison food (pathogenic bacteria and molds) and microorganisms with positive role in food production (as the model yeast Saccharomyces cerevisiae). The antimicrobial activity is surveyed for a period of 4 days, in order to analyze the remanence of the antimicrobial action of the EOs.

\section{Experimental}

\section{Plant materials}

Dried aerial parts of Ocimum basilicum L. (basil), Mentha piperita L. (peppermint), Origanum vulgare L. 
(oregano), were used for extraction the volatile oils. Arial parts from all this herbs were collected from Sibiu County, Romania in 2018 and they were dried in the shade.

\section{Essential oils extraction and characterization}

For obtaining and dosing the EOs, aerial parts were used. Extraction was realized through steaming for $5 \mathrm{~h}$ by using a neo-Clevenger equipment modified as by Moritz. The content was compared to the moisture less vegetal material [17].

The volatile oils were analyzed through by GC-MS. The analysis was performed with a Hewlett Packard 5890 III gas chromatograph equipped with a mass detector MS 5972. The chromatographic column used was a HP5-MS capillary column made of quartz, with a non-polar stationary phase consisting of $95 \%$ methyl and $5 \%$ phenyl polysillox. The constructive characteristics of the column are: length, $30 \mathrm{~m}$, interior diameter, $0.25 \mathrm{~mm}$ and thickness of the stationary phase, $0.25 \mu \mathrm{m}$. Helium was used as carrier gas $(1: 1 \mathrm{ml} / \mathrm{min})$. The injection temperature was $60{ }^{\circ} \mathrm{C}$, incrementing with $3 \mathrm{C} / \mathrm{min}$ to 240 ${ }^{\circ} \mathrm{C}$. To identify the separated components, an integrated GC-MS software was used. Library Wiley 275, used to recognition of chemical compounds was carried out by comparing the acquired mass spectra with mass spectra of standards program [17].

\section{Determination of the antimicrobial activity}

Antimicrobial action of the EOs was investigated on three types of microorganisms: pathogenic bacteria (Salmonella anatum ATCC 9270 and Bacillus cereus ATCC 10876), a mould contaminating food (Aspergillus niger ATCC 16404) and Saccharomyces cerevisiae ATCC 32701, a model non-pathogenic yeast. Before the analysis, the pure cultures of bacteria were maintained in Mueller-Hinton broth (MHB, Difco) for $48 \mathrm{~h}$ at $45{ }^{\circ} \mathrm{C}$, the pure culture of yeast were maintained in nutrient broth (BD Difco Nutrient broth) for $120 \mathrm{~h}$ at $20^{\circ} \mathrm{C}$ (yeast); the liquid broth was used. The mould was cultivated first on malt agar (Difco) for 5 days at $20{ }^{\circ} \mathrm{C}$, then the spores were harvested in nutrient broth and the liquid was used for the analysis.

Agar diffusion method was used to investigate the antimicrobial action of a quantity of $0.5 \mu \mathrm{l}$ of essential oil against microorganisms cultivated on nutrient agar (Difco Nntrient Agar); the inhibition percentage on the continuous film formed by the microorganism was determined by reporting the inhibition zone to the Petri dish area [18]. Five trials in parallel for each microorganism were made; the results were expressed as median value of all measurements.

Because the EOs are volatile, the antimicrobial activity was analyzed after $48 \mathrm{~h}, 72 \mathrm{~h}$ and $120 \mathrm{~h}$ in order to investigate the remanence of the antimicrobial action in time.

\section{Results and discussion}

EOs extracts from basil and peppermint have a white-yellowish color and the oregano oil has a yellow dark color. All EOs have characteristic flavors, slightly aromatic. The extraction yields are $0.66 \%$ for Ocimum basilicum $L$. volatile oil, $0.72 \%$ for Mentha piperita $L$. volatile oil, $0.16 \%$ for Origanum vulgare volatile oil.

The GC-MS analysis shows that all volatile oils analysed have a high number of components. The major compounds of the volatile oils are presented in Table 1.

Twenty five components in the volatile oil extracted from Ocimum basilicum L. were separated and identified through GC-MS. Eugenol and apiol are the major compounds and mean components are alcohols. 19 components in the volatile oil extracted from Origanum vulgare $L$. were separated and identified through GCMS; timol, $\gamma$-terpinene, and p-cymene are the major compounds. 26 components in the volatile oil extract from Mentha piperita $L$ were separated and identified through GC-MS; the main components of EO from peppermint are menthol and menthone.

Table 1 presents the results obtained at the analysis of the antimicrobial activity of all three EOs on the chosen microorganisms. Basil essential oil is having antimicrobial action on all microorganisms. In the first $48 \mathrm{~h}$ of analysis, the action decreased in the order: S. anatum (48\%), A. niger (26), B. cereus $(21 \%)$ and S. cerevisiae $(15 \%)$; this action is maintained after $72 \mathrm{~h}$ or $120 \mathrm{~h}$ for all samples. In the first $48 \mathrm{~h}$, the peppermint oil shows the highest inhibition (50\%) on B. cereus, followed by A. niger (45\%) and S. cerevisiae (20\%) and no inhibition on Salmonella; this action varies after $72 \mathrm{~h}$ and $120 \mathrm{~h}$ by slightly increasing in case of B. cereus, strongly decreasing (to $20 \%$ ) in case of A. niger and remaining constant in case of $\mathrm{S}$. cerevisiae. Oregano oil has the smallest antimicrobial action, only on S. anatum $(12 \%)$ and this action is maintained for $120 \mathrm{~h}$.

Scientific literature the differences in the extraction quantities and yields, due to variation in quantity, but also growing conditions (climate, soil constituents), part of the plant used for extraction, stage of ripening process. Also, the scientific information about EOs obtained from these plants are very different. For example, in case of EO extracted from American basil, the extraction yield varied between $0.07 \%$ and $1.92 \%$ and eugenol content was much smaller (5-29 \%) [19], compared with our results.

Regarding the peppermint EO, menthol and menthone were found by other authors, too, as the major components of the peppermint EO [20], whereas other researchers identified menthol, menthyl acetate and menthofurane as major compounds [11]. The extraction yield was found to be around $1 \%$ by other authors [21], higher as the content obtained by us, but a real comparison is difficult to be made because of the high number of variables influencing the yield.

Oregano essential oil yield (ranged from $0.114 \%$ to $2.3 \%$, depending on the distillation time and carvacrol was the main component in other researches, too [22].

The highest action against $S$. anatum showed by the basil essential oil (Fig. 1a), combined with the very low inhibition action of the oregano EO is in agreement with other researches [23]. The EOs chemical component in basil with the inhibitory action is eugenol causing the increasing of permeability by decreasing the membrane integrity, with the Minimal Inhibitory Concentration MIC of $0.025 \%$ against $S$. typhimurium [24]. 
Table 1. Major components of the volatile oils analysed

\begin{tabular}{|c|c|c|c|}
\hline Common name & Latin name & Major components & Composition \% \\
\hline \multirow{7}{*}{ Basil } & \multirow{7}{*}{ Ocimum basilicum $L$. } & Eugenol metyl eter & 41.55 \\
\hline & & Apiol & 14.23 \\
\hline & & Caryophyllene oxide & 7.83 \\
\hline & & Tau Cadinol & 7.47 \\
\hline & & Estragole & 6.62 \\
\hline & & Methyl cinnamate & 6.12 \\
\hline & & Linalool & 1.74 \\
\hline \multirow{3}{*}{ Peppermint } & \multirow{3}{*}{ Mentha piperita $L$. } & Menthol & 49.5 \\
\hline & & Menthone & 21.9 \\
\hline & & Limonene & 5.80 \\
\hline \multirow{4}{*}{ Oregano } & \multirow{4}{*}{ Origanum vulgare $L$. } & Tymol & 55.25 \\
\hline & & $\mathrm{y}$-terpinene & 15.22 \\
\hline & & p-Cimen & 6.28 \\
\hline & & Carvacrol & 3.31 \\
\hline
\end{tabular}

As $[25,26]$ indicated, peppermint EO has a weak antibacterial activity. The result obtained at the analysis of the inhibitory analysis of peppermint oil on S. anatum is in accordance with [25], whereas [16] indicated a weak inhibitory action of peppermint oil against $S$. typhimurium, with menthol as the main component with inhibitory activity.

Peppermint EO affects mostly the other three microorganisms investigated, as figures $1 \mathrm{~b}, 1 \mathrm{c}$ and $1 \mathrm{~d}$ show. Bacillus cereus is the most affected by peppermint essential oil; this result is in accordance with literature [27]. The antifungal action of peppermint oil against $A$. niger is correlated with the main component menthol, as [30] indicated. Other research reported the strong inhibitory action of the peppermint oil against $S$. cerevisiae [28], but in our case this action was moderate; anyway, the EO seems to affect the cell membrane, as in the case of basil essential oil.

In [28] indicates that the growth of the yeast $S$. cerevisiae seems to affected by oregano oil (even in much smaller percentage as by the peppermint oil.

In [29] shows the strong influence of the oregano oil on $A$. niger. Despite its content in carvacrol, a very efficient antimicrobial compound [30], oregano essential oil extracted by us has a slightly action against $S$. anatum and no action against the other microorganisms studied here. Probably the concentration tested here is too small for inhibiting the microbial growth.

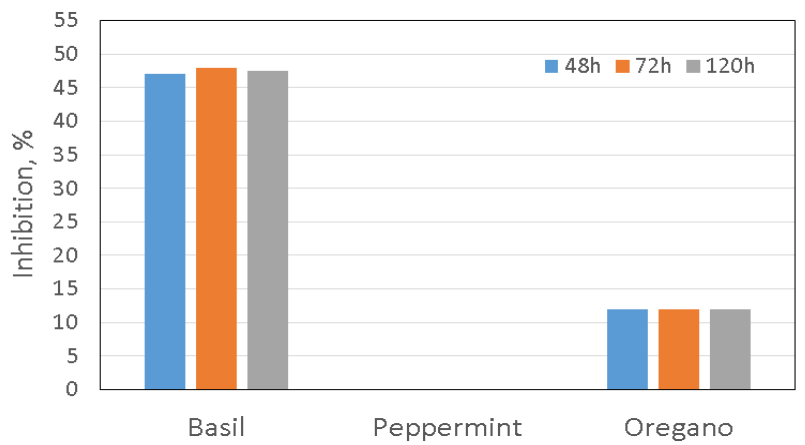

a) Salmonella anatum

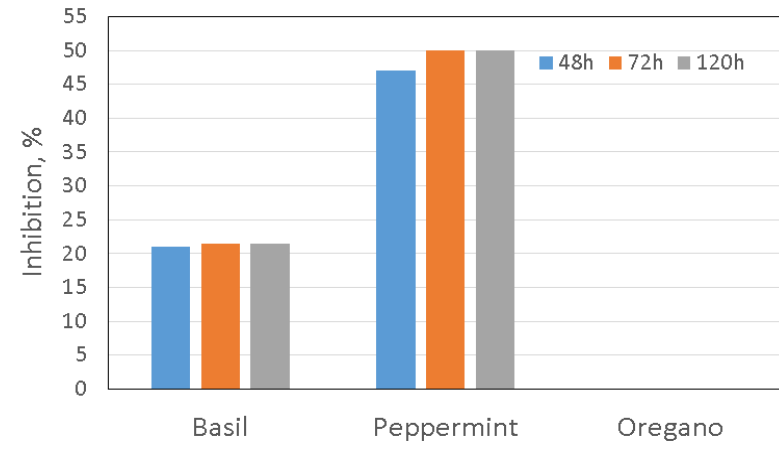

b) Bacillus cereus

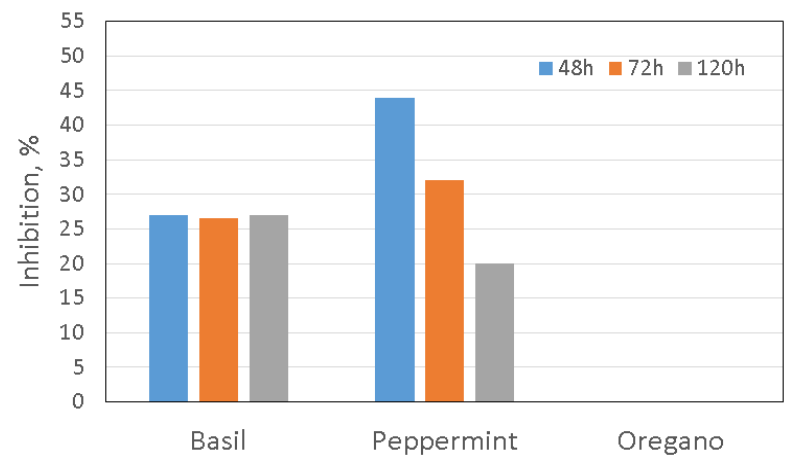

c) Aspergillus niger

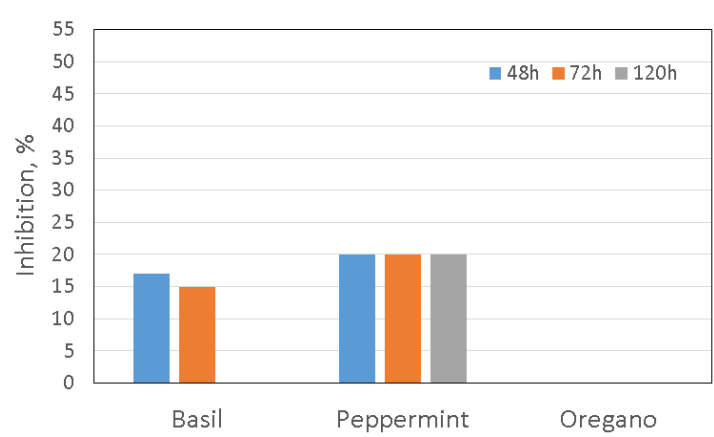

d) Saccharomyces cerevisiae

Fig. 1. Inhibition action of basil, peppermint and oregano essential oils against Salmonella anatum, Bacillus cereus, Aspergillus niger and Saccharomyces cerevisiae 


\section{Conclusion}

EOs were extracted from basil, peppermint and oregano leafs in the Sibiu County, Romania with good yields, comparable with other similar plants from other regions of the world. Essential oils have a valuable composition in eugenol, menthol and carvacrol, three very efficient antimicrobials; they were found in high amounts in plants.

EOs showed different antimicrobial action on the four microorganisms tested at the concentration tested $(0.5 \mu \mathrm{l})$, in the general order: peppermint oil $>$ basil oil $>>$ oregano oil; this action remained quite constant for $120 \mathrm{~h}$. The most sensitive to basil oil was $S$. anatum.

\section{References}

1. E.Christaki, E. Bonos, I. Giannenas, and P. FlorouPaneri, Agriculture, 2, 228-243 (2012)

2. W. Dhifi, S. Bellili, S. Jazi, N. Bahloul, and W. Mnif, Medicines, 3(4), 25 (2016)

3. F. Bakkali, S. Averbeck, D. Averbeck, and M. Idaomar, Food Chem Toxicol, 46, 446-475 (2008)

4. Y. Seow, C. Yeo, H. Chung, and H.G. Yuk, Food Science and Nutrition, 54(5), 625-644 (2013)

5. S.M. Sharafi, I. Rasooli, P. Owlia, M. Taghizadeh, and S.D.A. Astaneh, Pharmacogn Mag., 6(23), 147 153 (2010)

6. Y. Kumar, O. Prakash, H. Tripathi, S. Tandon, M. M. Gupta, L.U. Rahman, R.K. Lal, M. Semwal, M. P. Darokar, and F. Khan, Frontiers in Plant Science, 9, 108 (2018)

7. C.S. Sell, The chemistry of fragrance. From perfumer to consumer, 2nd ed., (The Royal Society of Chemistry, Cambridge, UK, 2006)

8. L.A. Conde-Hernández, J. R. Espinosa- Victoria, A. Trejo, and J. Guerrero-Beltrán, Journal of Food Engineering, 200, 81-86 (2017)

9. A. ElAsbahani, K. Miladi, W. Badri, M. Sala, E.H. AitAddi, H. Casabianca, A. ElMousadik, D. Hartmann, A. Jilale, F.N.R. Renaud, and A. Elaissari International Journal of Pharmaceutics, 483 (1-2), 220-243 (2015)

10. D. Stojković, J. Glamočlija, A. Cirić, M. Nikolić, M. Ristić, J. Siljegović, and M. Soković, , Arch. Biol. Sci., 65(2), 639-643 (2013)

11. M.J. Saharkhiz, M. Motamedi, K. Zomorodian, K. Pakshir, R. Miri, and K. Hemyari, "Chemical composition, antifungal and antibiofilm activities of the essential oil of Mentha piperita L.", ISRN Pharmaceutics (2012)

12. A.R. Koroch, J.E. Simon, and H.R. Juliani, "Industrial Crops \& Products", 107, 526-530 (2017)

13. G. Iscan, N. K lrimer, Mn. Kürkcüoglu, H.C. Baser, and F. DeMirci, J Agric Food Chem., 50(14), pp. 3943-3946 (2002)

14. S. Rubab, I. Hussain, B.A. Khan, A.A. Unar, K.A. Abbas, Z.H. Khichi, M. Khan, S. Khanum, K.U.
Rehman, and H. Khan, J. Islam Int Med Coll, 12(1), 59-67 (2017)

15. A. Coelho da Costa, B.E. Cavalcanti dos Santos, F.L. Santos, and E.O. Lima, Rev. Bras. Farmacogn., 19, 236-241 (2009)

16. L. Tommasi, C. Negro, A. Miceli, and F. Mazzotta, J. Essent. Oil Res, 21, 185-189 (2009)

17. C. Georgescu, and M. Mironescu, Journal of Environmental Protection and Ecology 12, 4A, 2294-2302 (2011)

18. L.I. Virchea, C. Georgescu, and M. Mironescu, Obtaining and characterization of volatile oils from aromatic plants in 1st International Symposium Innovations in Life Sciences, 18-19 October, 2019, Belgorod, Russia (2019)

19. V. Zheljazkov, A. Callahan, and C. Cantrell, J Agric Food Chem, 56(1), 241-245 (2008)

20. J. Dai, V Orsat, G.S.V. Raghavan, and V. Yaylayan, Journal of food engineering, 96, 540-543 (2010)

21. J. Scavroni, C. S. F. Boaro, M.O.M. Marques, and L.C. Ferreira, Braz. J. Plant Physiol., 17(4), 345352 (2005)

22. V. D. Zheljazkov, T. Astatkie, and V. Schlegel, HortScience, 47(6), 777-784 (2012)

23. V.K. Bajpai, K-H. Baek, and S.C. Kang, Food Research International, 45(2), 722-734 (2012)

24. K P. Devi, S. A. Nisha, R. Sakthivel, and S. K. Pandian, Journal of Ethnopharmacology, 130(1), 107-115 (2010)

25. K. Wińska, W. Mączka, J. Łyczko, M. Grabarczyk, A. Czubaszek, A.Szumny, Molecules, 24(11), 2130 (2019).

26. H.W. Lim, D.H. Kim, S.H. Kim, J.M. Lee, J.W. Chon, K.Y. Song, D. Bae, J. Kim, H. Kim, and K.H. Seo, Journal of Milk Science and Biotechnology, 36(3), 146-154 (2018)

27. M. Moghtader, Academic journals, 7(11), 521-527 (2013)

28. H.B. Konuk and B. Ergüden, Biocell, 41(1), 13-18 (2017)

29. C. Chaves-López1 A. M. Martin-Sanchez, E. Fuentes-Zaragoza, M. Viuda-Martos, J. FernándezLópez, E. Sendra, E. Sayas, and J. A. Pérez Alvarez, J Food Prot, 75(1), 104-111 (2012)

30. A. Ben Arfa, S. Combes, L. Preziosi-Belloy, N. Letters in Applied Microbiology, 43, 149-154 (2016) 